\title{
Research on variant design based on topological entity compression algorithm
}

\author{
Yulin Shuai $^{\text {a }}$, QiuJiao Wang, and Juan Chen \\ School of Mathematics, Southwest Jiaotong University, Chengdu 611756, China
}

\begin{abstract}
In order to improve the efficiency of product design and shorten the design cycle of product, the research progress in recent years is introduced. The basics of variant design such as the entities of topology, the equation's structure and the compression/decompression are analyzed. An algorithm, which can compress redundant parts, based on the conditions of algebraic equations completely is put forward. A variant design system of volumetric heat exchanger is designed by using VB and SolidWorks API (Application Programing Interface). The results show that the variant design system based on algebraic equations can be quickly and accurately modeled according to different process parameters, which can improve the efficiency of product design and shorten the design period.
\end{abstract}

\section{Introduction}

In 3D graphics, entity refers to all objects composed of visual graphics, such as point, line and surface elements, stretching, rotation and other elements, product components, with the topology relationship between various entities. The current mainstream design software of CAD (Computer Aided Design) to the present and the design of visual graphic, such as CATIA SolidWorks and Pro/e are using the way of topological entities. The variant design refers to the basic principle of the product does not change, only through specific parameters changing entities or different entities topological relation, and the rapid design of new products [1-2].

Qin chao ruan[3],Li fen liang [4] have studied the variant design based on parameters configuration, by giving some parameters multiple discrete values, establishing different parameters, Form different configurations of an entity. When the technological parameters are changed, selecting the corresponding configuration of physical parameters to change the parameters. However, the method can only assign discrete values to the entity parameters and parameter values can't be changed continuously. Chen qiang luo [5] of the variant design based on the two development of the way, mainly through the $\mathrm{VB}, \mathrm{C}++$ and other high-level programming language called 3D software driver software of API drawing, can achieve larger. But the method involves variant design function of API, many built-in the complex structure and long development cycle.

The variant design based on algebraic equation can establish algebraic equation in different parameters, which can avoid the complicated programming, and can make the parameters continuous change, can avoid the deficiency of the 2 kinds of variant design., Gao Jie[6] ,Song Long [7] of variant design based on algebraic equations, greatly improves the design efficiency of graphics. But only in the entity hierarchy which topological entities are not involved in assembly level compression. 
In this paper, based on the document [6-7], under the SolidWorks software environment, a topology entity compression algorithm is proposed, and the variant design based on topology entity compression is studied at the assembly level.

\section{Solidworks orks variant design basis}

\subsection{Entity topology structure}

The SolidWorks' entities topology structure in general as shown in Figure 1, the $\mathrm{S}$ assembly contains a sub assembly S1 ,S2 and nesting child assembly S3, and the component P1, P2. The cooperation parts of components belongs to the two common parent file [8], such as with the relationship between $\mathrm{P} 1$ and $\mathrm{P} 2$ which belong to S3, if $\mathrm{P} 1$ move up one level, and tied with S3, it will move to $\mathrm{S} 1$; if the P1 moved to $\mathrm{S} 2$, with the relationship will move to the top assembly $\mathrm{S}$.

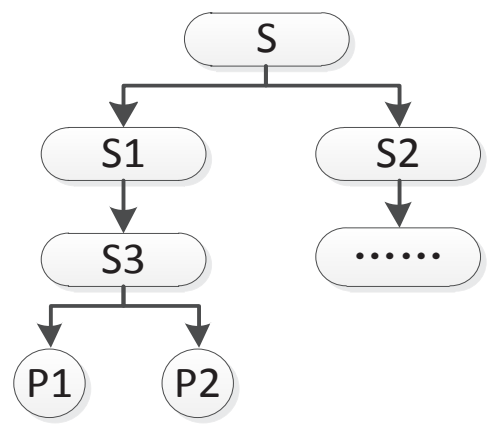

Figure. 1

\subsection{Assembly equation structure}

In SolidWorks assembly, the commonly used equation forms are mainly 3 kinds of "global variables", "characteristic" and "equation - Parts", as shown in Figure2.

"Global variables" refers to change the underlying active parameters of the parameters, the representation is $\mathrm{A}=\mathrm{P}$; "features" for the compression or decompression parts, the representation is $\mathrm{B}=\mathrm{S}$;" equation - Parts" refers to the size parameters of parts, the representation is $\mathrm{C} @ \mathrm{D} @ \mathrm{~B}-\mathrm{i}=\mathrm{P}$

Among
A -- Global variable name
B -- The name of the component

C -- Parameter names for parts sketches or features, such as length, width, and height

D -- Part sketch or feature name

$\mathrm{P}$-- The numerical of a variable or algebraic equation

$\mathrm{S}$-- Parts status. There are 2 states of compression and decompression

I -- Identification code of parts .In SolidWorks assembly will be used in multiple instances of a component, which is to add multiple parts to the same assembly, parts identification code for each instance to distinguish between parts, according to the order assignment is added to the assembly of $1,2,3 \ldots \ldots$.

Examples of the 3 representations are as follows:

$$
\begin{gathered}
\text { "Diameter" }=40 \\
\text { "Part1-1. part"=suppressed } \\
\text { "H@Sketch1@Part-1. Part"=unsuppressed }
\end{gathered}
$$

\subsection{Compression and decompression}

Compression of topological entities (suppressed) refers to the parts removed from the model in computer memory, and hidden from the assembly, but does not remove it, before relieving compression (unsuppressed), it makes temporarily unavailable. Compression can reduce the storage and memory, compression plays an important role in the graphic image file storage, transmission and processing.

There are 2 general ways of compression or decompression of an entity, unconditional execution command, such as the above formula gives the suppressed or unsuppressed command directly on the right end; second is the condition of compression, that meet compression or compression of entities will be existed if certain conditions satisfied.

\section{"Part2-1. Part"=IF ("Diameter" \\ $<50$, suppressed, unsuppressed)}

In this paper, an algorithm for condition compression of topological entities is proposed. The components used in the previous stage are added to the assembly, and the constraints are set. The input condition is used to determine whether to compress or decompress of the entity.

\section{Heat exchanger structure}




\subsection{Composition of heat exchanger}

Volume type heat exchanger is a key product of the centralized water supply system, widely used [10] with the water storage capacity is large. Its shape as shown in Figure 3, which is mainly composed of a cylinder body 1, cylinder head 2, the heat exchanger core component 3 (including tube box, flange head, etc.), hot import and export component 4 , the refrigerant components import and export group5, tube box and outlet6, lug 7, pressure gauge8, instrument tube 9, emission components 10 , base 11 and other parts. There are a large number of driven parameters, whether early design or late manufacturing engineering drawings, all need to spend a lot of time, variant design of its products is necessary.

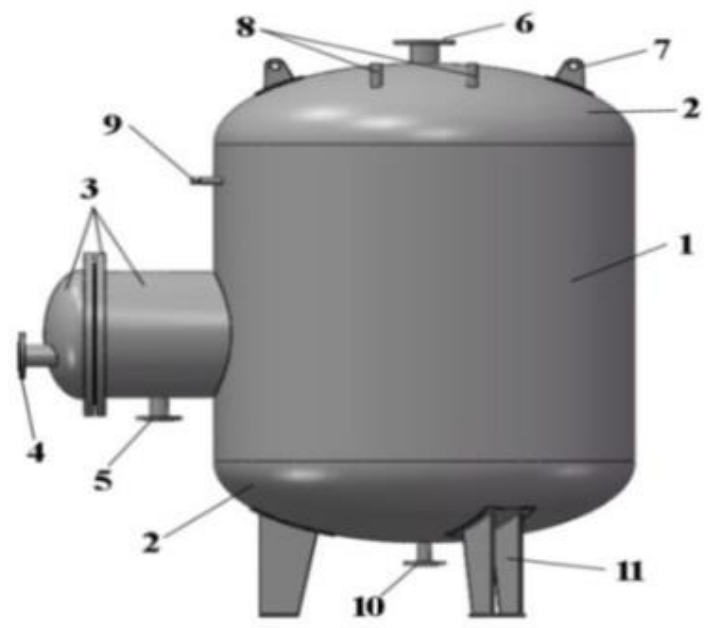

Figure. 3

\subsection{Ellipsoidal head}

The head of heat exchanger is usually ellipsoidal head, whose plane projection is elliptical, and the semi long axis $a$ and half short axis $b$ meet $a=2 b$

Elliptical semi long axis a and barrel diameter D meet $\mathrm{a}=\mathrm{D} / 2$

As shown in Figure 4, the $\mathrm{OA}$ is $\mathrm{X}$ axis, the $\mathrm{OB}$ is $\mathrm{Y}$ axis, establishment of coordinate system, and safety valve pipe length is $L_{0}$, coordinates of $\mathrm{M}$ with the highest point branch need to obtain, and then taking $\mathrm{M}$ as the starting point down the stretch entity to the exchanger head.

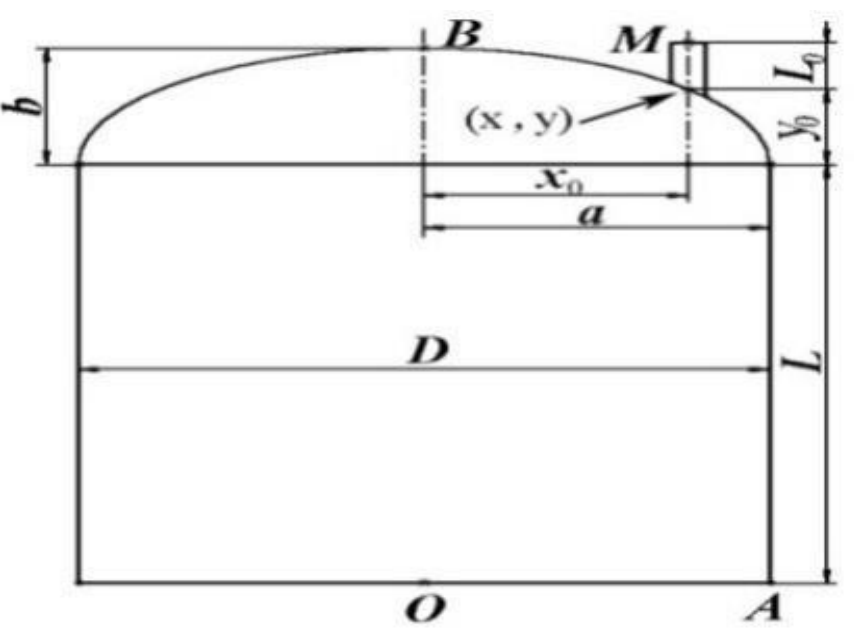

Figure. 4

The distance between one point of the ellipse (x, y) and the long axis and the short axis is $y_{0}$ and $x_{0}$ respectively, and the two relations satisfy the relation

$$
y_{0}=\frac{b}{a} \sqrt{\left(a^{2}-x_{0}^{2}\right)}
$$

The longitudinal coordinate $y_{M}$ of the branch starting point $M$ is satisfied

$$
y_{M}=L+y_{0}+L_{0}
$$

The abscissa $x_{M}$ is satisfied with the diameter of the cylinder $D$ :

$$
x_{M}=k D
$$

Among them, $\mathrm{k}$ is the constant factor .

By combining the above formula with figure 5, we can calculate the relation between the ordinate $y_{M}$ and the global variable $D$.

$$
y_{M}=L+\frac{D}{4} \sqrt{\left(1-4 k^{2}\right)}+L_{0}
$$

The relation is expressed as follows in the SolidWorks algebraic equation:

"Ordinate@Branch@Barral-1.Part"=

"Length"+" Diameter" / ${ }^{*} \operatorname{sqr}(1-4 * k * k)+L_{0}$

The designer can evaluation $L_{0}$ and $k$ according to 
the specific process requirements.

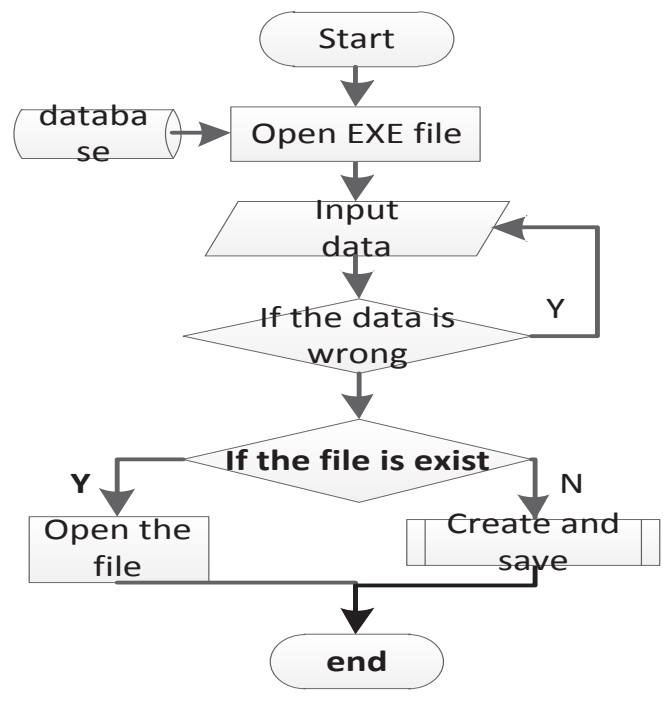

(a)

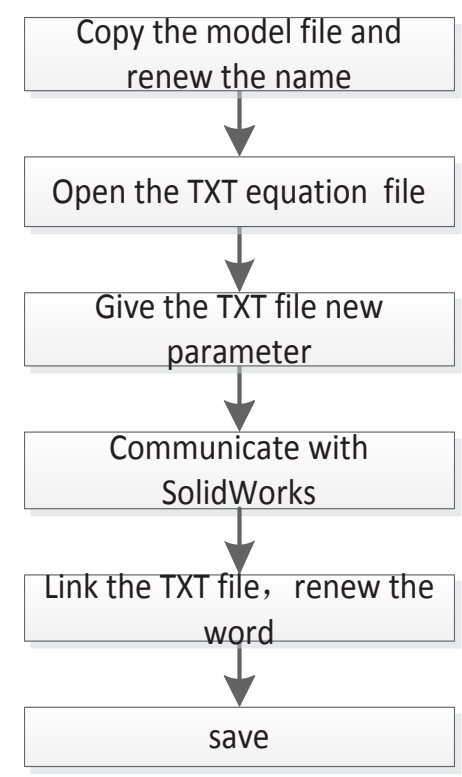

(b)

Figure.5

\section{Conditional compression}

\section{algorithm for topological entities}

\subsection{Algorithm flow}

Although there are quite a number of the heat exchanger parameters, most of the parameters are driven parameters. Using the variant design based on algebraic equations, the parameter setting can be active in the global variable, then driven parameters and establish algebraic equations. When the parameters change, it can only modify the active parameters, all driven parameters associated with changes so that form the new products.

The algebraic equation can be used with external TXT text file format for data transmission. The programming language generated EXE file, modify the TXT file in the form of expression data to modify global variables and equations. Using API in swEqnMgr: FilePath links graphics files and TXT files for external.

The algorithm flow chart in figure 5- (a), which including the new sub process and save the document in figure 5- (b). If the model file of the required parameters is already exists, then open the file directly. If the file does not exist, the TXT data file is the first copy the model files and links, and named the model file as an input parameter,it is easy to search; and then open the TXT file, saving the TXT document after modify the parameters corresponding to the value of the global variable; finally open model file reconstruction (refresh) model and save. Modify global variables, all the equations solved automatically.

\subsection{Main code of algorithm}

To achieve the above algorithm Visual Basic program, the core code is as follows:

DocName $=$ "RH"

$\mathrm{N}=$ combo.UBound

For $\mathrm{i}=1$ To N

DocName = DocName \& "-" \& combo (i) ' Use the file's own parameters to name the file

Next i

DocPath = App.Path \& DocName \& ".SLDASM" ' File (assembly) path

If $\operatorname{Dir}($ DocPath) $<>$ "" Then ' Determine if the file already exists

Msgbox "The file already exists!"

Else ' If it does not exist, new file

Open App.Path \& "\RH.txt" For Input As \#1 'Open

...... 'Change the original parameter to the new parameter and write to the TXT file

Set SWAPP $=$ Create Object ("SldWorks. Application") '

Communicate with SolidWorks

Set Model = SWAPP.NewDocument (App. Path \& "l" $\&$ DocName $\&$ ".SLDASM", 0, 0,0) 'Open file 
Set swEqnMgr $=$ Model.GetEquationMgr swEqnMgr.FilePath = App.Path \& "।" \& DocName \& ".txt" ' Link external TXT files

boolstatus $=$ Model.EditRebuild3() ' Reconstruction model

End if

\subsection{Examples of conditional contraction of topological entities}

The nominal diameter of the conventional volumetric heat exchanger is generally $800 \mathrm{~mm} \sim 1600 \mathrm{~mm}$, and the nominal diameter of the outlet of the heat exchanger with different parameters is selected in the set $Q=\{32,40,50,65,80\}$, and the nominal diameter of the flange is also one of the 5 parameters mentioned above

At the beginning of the design template, the flanges of the above 5 parameters are added to the assembly and are exactly the same as the drain pipe. Then the following equations are set

"Flange name-identification code. Part"

=IF ("Outlet diameter"=Specific parameters For

, unsuppressed, suppressed)

example, the flange is named "FL- nominal diameter", and the identification code for the discharge flange is 2 .

The algebraic equation for the flange with nominal diameter $32 \mathrm{~mm}$ is shown as follows

"FL-32-2. Part"=IF ("Outlet diameter"

=32, unsuppressed, suppressed)

When the nominal diameter of the discharge pipe is equal to $32 \mathrm{~mm}$, the flange is decompress. Otherwise it has been in a compression state. Thus, the flange assembled with the discharge pipe will change accordingly with the change of the parameters of the sewage pipe.

\subsection{Multiple conditional judgment}

For parameters that vary in gradient according to global variables, the IIF function can be used to establish equations for handling multiple conditions, such as the height of the bearing and the nominal diameter, which correspond to the relation shown in Table 1.

Table 1

\begin{tabular}{|c|c|}
\hline $\begin{array}{c}\text { Nominal diameter of heat } \\
\text { exchanger }\end{array}$ & Bearing height \\
\hline $800 \sim 1000$ & 350 \\
\hline $1100 \sim 1400$ & 420 \\
\hline $1500 \sim 1800$ & 460 \\
\hline
\end{tabular}

For the height of the support, write the algebraic equations as shown below so that they vary automatically according to the input parameters.

$$
\begin{aligned}
& \text { "h@H@Part-1.Part" } \\
& =I I F(" \text { Diam"<=1000,350, } \\
& \text { IIF("Diam" }<=1400,420,460))
\end{aligned}
$$

\subsection{Algorithm analysis}

The variant design provided the mainstream CAD the design software, variant design based on the parameter configuration only give to the entity parameters discrete values ,parameter values can't be changed continuously, the latter use is restricted, the life cycle of variant design system is short, the preliminary design is mainly applied to small variant entities; variant design based on API develop of two times need to use advanced programming language called software's API, and the API number is large, complex structure, the long development cycle.

The proposed algorithm based on topological entity compression between the parameters of different parts established algebraic equation, the parameter changes continuously, no parameter group can significantly extend the discrete additions, prolong the system life cycle; only by programming in terms of parameter transfer and interactive interface, avoids the complex API programming, greatly shorten the system design cycle. In addition, the algorithm of top-down, variant design with global in the assembly level, is suitable for the sketch, parts and assembly, also avoids the shortcomings of the algorithm only applies to parts of entities in the literature [6-7].

\section{Conclusion}


A algorithm based on redundant topology entity conditions compression, the study of variant design in assembly hierarchical top-down, parameters in different parts were correlated by algebraic equations. Using VB language to implement the algorithm, and design the heat exchanger variant design system. The variant design system base on topological entities compressed can quickly and accurately process variant design according to different parameters, which can avoid the complex programming and a large number of parameters calculate, and can make the parameters change continuously, improve the efficiency of product design, shorten the design cycle of the system.

This work was partially supported by the Basic

Research Fund of Central University

(No.2682014ZT28).

E-mail addresses:1021734635@qq.com(Y.Shuai)

\section{Reference}

[1]T. T. qian,Study on variant design method and application of hydraulic support [D]. Suzhou: Suzhou University, (2015).

[2]C.G. Yao, Y. H. Sun, B.Kuang, et al. Variant design of assembly parts based on parametric technology [J]. Computer system applications,21,(2012), 160-163.

[3]Q.C.Ruan, W. P.Dong. Parametric modeling of SolidWorks based wedge mechanism [J]. Journal of Shanghai University of Engineering Science,
30(2016), 149-154.

[4]L.F.Liang, Z.Y.Wang,S.F.Wu, et al. Parametric design of reducer case structure based on SolidWorks configuration technology [J]. Mechanical transmission,39 (2015), 80-83.

[5]C.Q.Luo,L.W.Zhong,J.Zhu,Rapid design system of crane lifting mechanism based on configuration technology [J]. Computer system applications, 21 (2012) ,18-21.

[6]J.Gao, Research and development of construction hoist CAD system based on [D]. Solidworks Xi'an: Chang'an University, (2013).

[7]L.Song,L.Ma,Z.X,Jia. Research on parametric technology of drive mechanism of crank press based on [J].. SolidWorks forging technology, $\mathbf{4 0}$ (2015), 107-111.

[8]SolidWorks ${ }^{\circledR}$ company SolidWorks part and assembly tutorial [M]. Beijing: Mechanical Industry Press,(2014): 328-394.

[9]C. C.Wang,L.J.Wang,Y.Z.Zhao, et al. Study on automatic assembly technology of Modular Parallel Robot Based on [J].SolidWorks mechanical design and manufacture, (2009), 166-168.

[10]C.Lu,D. Z.Zhu. The experimental study on water supply by coil and volumetric heat storage water tank [J]. Journal of solar energy,29(2008), 569-573

\begin{tabular}{|l|l|l}
\hline Name & Value / Equation & Evaluates to - \\
\hline$\square$ Global Variables & & \\
\hline "Diameter" & $=40$ & 40 \\
\hline Add global variable & & \\
\hline- Equations-Components & & \\
\hline "D2@Sketch5@DZ-1100-10-1.Part" & $={ }^{-D i a m e t e r " / 2 ~}$ & $20 \mathrm{deg}$ \\
\hline Add equation & & \\
\hline- Dimensions - Top Level & & \\
\hline D3@LocalCirPattern1 & $180 \mathrm{deg}$ & $180 \mathrm{deg}$ \\
\hline D1@LocalCirPattern1 & 2 & 2 \\
\hline D1@Distance1 & $200 \mathrm{~mm}$ & $200 \mathrm{~mm}$
\end{tabular}

Figure.2 